\title{
Design and methods of the Population Assessment of Tobacco and Health (PATH) Study
}

\author{
PATH Study Team \\ - Please see end of paper for full author list
}

\section{Correspondence to}

Dr Andrew Hyland, Department of Health Behavior, Roswell Park Cancer Institute, Buffalo, NY 14263, USA;

Andrew.Hyland@roswellpark. org

Received 12 January 2016 Revised 14 July 2016 Accepted 15 July 2016 Published Online First 8 August 2016
CrossMark

$$
\begin{aligned}
& \text { To cite: PATH Study } \\
& \text { Team. Tob Control } \\
& \text { 2017;26:371-378. }
\end{aligned}
$$

\section{ABSTRACT}

Background This paper describes the methods and conceptual framework for Wave 1 of the Population Assessment of Tobacco and Health (PATH) Study data collection. The National Institutes of Health, through the National Institute on Drug Abuse, is partnering with the Food and Drug Administration's (FDA) Center for Tobacco Products to conduct the PATH Study under a contract with Westat.

Methods The PATH Study is a nationally representative, longitudinal cohort study of 45971 adults and youth in the USA, aged 12 years and older. Wave 1 was conducted from 12 September 2013 to 15 December 2014 using Audio Computer-Assisted SelfInterviewing to collect information on tobacco-use patterns, risk perceptions and attitudes towards current and newly emerging tobacco products, tobacco initiation, cessation, relapse behaviours and health outcomes. The PATH Study's design allows for the longitudinal assessment of patterns of use of a spectrum of tobacco products, including initiation, cessation, relapse and transitions between products, as well as factors associated with use patterns. Additionally, the PATH Study collects biospecimens from consenting adults aged 18 years and older and measures biomarkers of exposure and potential harm related to tobacco use.

Conclusions The cumulative, population-based data generated over time by the PATH Study will contribute to the evidence base to inform FDA's regulatory mission under the Family Smoking Prevention and Tobacco Control Act and efforts to reduce the Nation's burden of tobacco-related death and disease.

\section{INTRODUCTION}

It has been over 50 years since the Surgeon General of the USA first concluded that "cigarette smoking is a health hazard of sufficient importance in the United States to warrant appropriate remedial action". ${ }^{1}$ Following decades of additional research, the 2014 Surgeon General's Report (SGR) on the health consequences of smoking, ${ }^{2}$ which marked the 50th anniversary of the first SGR, identified additional diseases caused by smoking. Scientific evidence now supports that smoking causes at least 15 types of cancer, as well as numerous chronic diseases including heart disease, stroke, lung diseases and diabetes. ${ }^{2}$ The 2014 SGR highlighted the need for continued implementation of comprehensive tobacco control programmes and policies.

Cigarette smoking prevalence has been halved since publication of the 1964 SGR, yet tobacco use remains the leading preventable cause of disease and premature death in the USA. Over 480000 annual US deaths ${ }^{2}$ and a combined 14 million comorbid conditions in $2009^{3}$ were attributed to cigarette use and secondhand cigarette smoke exposure. Cigarette use continues to dominate the US tobacco market, despite the decrease in per capita consumption. ${ }^{2}$ However, the diversification of the US tobacco market has increased in recent years, and current patterns of tobacco use reflect the evolving marketplace. ${ }^{2}$ Findings from the 2013 National Survey on Drug Use and Health (NSDUH) indicate that an estimated 66.9 million Americans $(25.5 \%$ of the population aged 12 and older) used at least one tobacco product in the past 30 days; $\sim 55.8$ million persons $(21.3 \%$ of the population) smoked cigarettes; 12.4 million (4.7\%) smoked cigars; 8.8 million (3.4\%) used smokeless tobacco and 2.3 million $(0.9 \%)$ smoked tobacco in pipes. ${ }^{4}$ From 2011 to 2014, past 30-day use of e-cigarettes and hookah (also known as 'waterpipe') increased among youth, whereas cigarette and cigar smoking declined. ${ }^{5}$ Recent findings from the National Youth Tobacco Survey (NYTS) and the Monitoring the Future (MTF) study indicate that e-cigarette use now exceeds cigarette smoking among youth. ${ }^{5} 6$

On 22 June 2009, the landscape changed dramatically for tobacco use prevention and control in the USA when the Family Smoking Prevention and Tobacco Control Act (TCA) was signed into law. ${ }^{7}$ The TCA gave the Food and Drug Administration (FDA) broad regulatory authority over the manufacturing, marketing and distribution of tobacco products $^{\mathrm{i}}$ to protect the Nation's health. This authority includes, but is not limited to, developing product standards; enforcing youth access restrictions, including restrictions on marketing, advertisements, promotions and sales to youth; authorising marketing orders for new or modified risk tobacco products and mandating the labelling of tobacco products, including larger and/or graphic health warnings on product packages and

iThe TCA amended the Federal Food, Drug and Cosmetic Act (FD\&C Act) to give FDA the authority over tobacco products-defined as products made or derived from tobacco intended for human consumption that are not otherwise regulated as a drug or a device (section 101[a] [rr][1]), including cigarettes, cigarette tobacco, smokeless tobacco and roll-your-own tobacco (section 901[b]). The TCA gave the HHS Secretary authority to deem authority over products meeting the statutory definition of a tobacco product. The FDA released its proposed rule to assert authority over other tobacco products that meet the definition of a tobacco product including, but not limited to, e-cigarettes, cigars and hookah on 25 April 2014 and can be found at http://www.gpo.gov/fdsys/pkg/FR-201404-25/pdf/2014-09491.pdf. 
in advertisements. In contrast to other Centres within the FDA that review therapeutic products using a 'safe and effective' standard, the FDA's Center for Tobacco Products (CTP) was charged with regulating tobacco products through a standard based on population health weighing potential benefits and harm to current, former and never users of tobacco products. Several models that operationalise the population health standard have been proposed. ${ }^{8-10}$ The Population Assessment of Tobacco and Health (PATH) Study was launched to generate longitudinal epidemiologic data on tobacco use behaviours, including patterns of use, attitudes, beliefs, exposures and health among the US population to inform, and to monitor the impact of FDA's regulatory actions under the Federal Food, Drug and Cosmetic Act to reduce tobacco-related death and disease.

\section{THE PATH STUDY CONCEPTUAL MODEL}

The PATH Study is based on the Host, Agent, Vector, Environment (HAVE) conceptual model (figure 1) that has been validated in assessing the impact of several tobacco control policy interventions. ${ }^{11}$ This model is often used to illustrate the transmission of infectious agents, but it has also been used for tobacco studies, including the International Tobacco Control (ITC) Project, which, like the PATH Study, follows nationally representative samples of respondents longitudinally to assess factors associated with tobacco use. ${ }^{12}$ Host factors are intrinsic to the individual; that is, to individual tobacco users or to those who are at risk of becoming tobacco users, including the individual's perceptions and social, demographic and genetic characteristics. Agent factors are specific to tobacco products, which can include features of product design and formulation as well as tobacco-product packaging and promotions. Environmental factors encompass policy changes, such as implementation of a product standard, as well as the broader social, cultural and geographic influences. The vector component is the vehicle (eg, tobacco manufacturers) that introduces, promotes and facilitates interactions of the agent (eg, tobacco products) with the host and environment to result in downstream behavioural and health outcomes. The HAVE model assumes that host, agent, vector and environmental factors interact to influence a variety of behavioural and health outcomes. The model does not posit a causal chain of events; rather, it provides a framework for how different types of measures relate to each other to impact the outcome of interest. ${ }^{13}$ For example, efforts to educate the public about the harm of tobacco products would be expected to raise awareness and influence risk perceptions, which in turn may increase cessation and decrease initiation activity, thereby improving health
Figure 1 Host, agent, vector and environment model applied to tobacco with examples of constructs within each domain. ${ }^{11}$

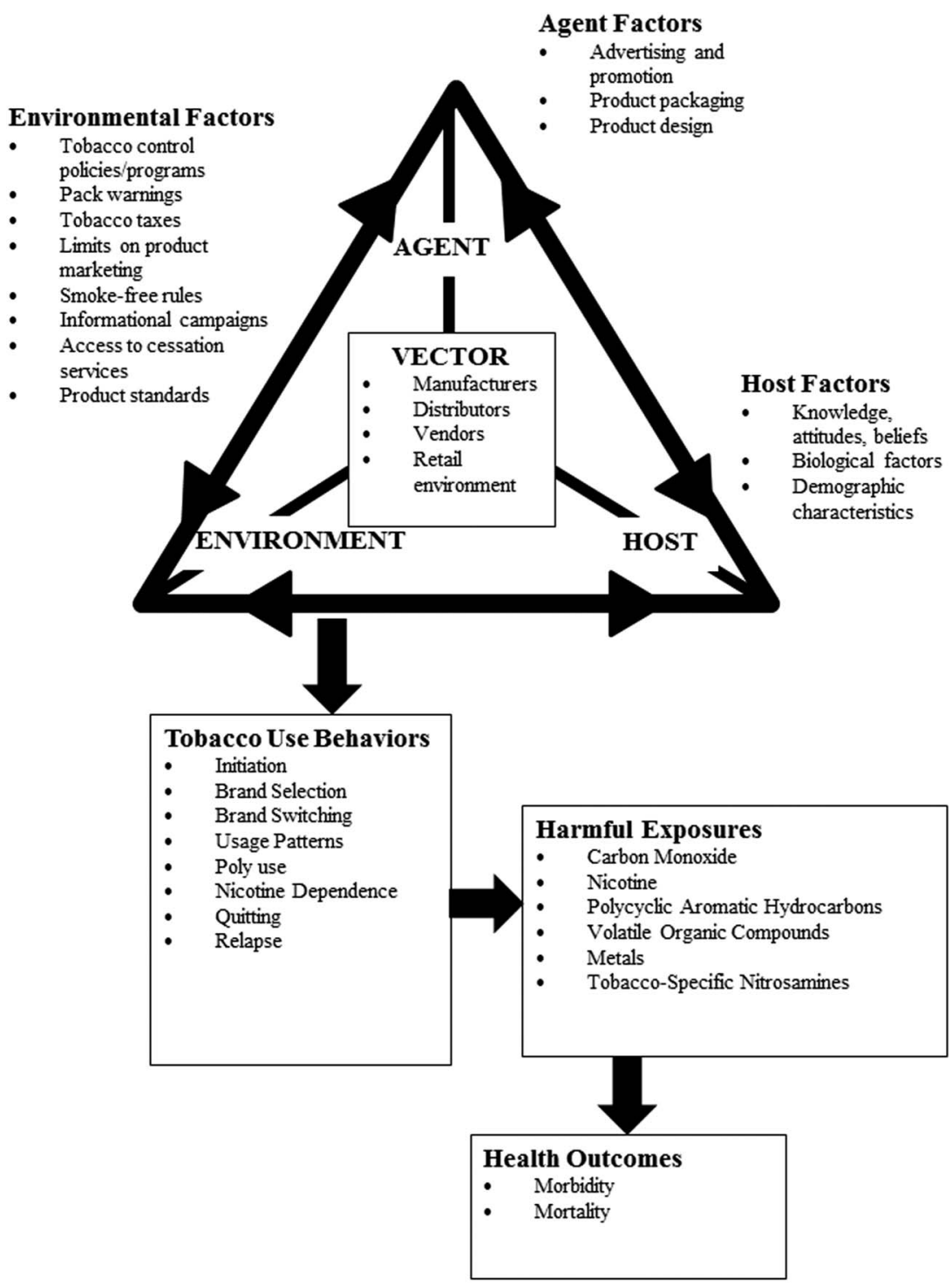


outcomes. In addition, the transdisciplinary approach to understanding tobacco use considers transitions and the factors that influence them iteratively, and cumulatively, over time, including factors specific to individuals (eg, attitudes, beliefs, biological factors) as well as external factors (eg, exposure to tobacco product marketing, tobacco policies). ${ }^{14-18}$

The PATH Study is a research study that assesses withinperson changes and between-person differences in a large national cohort of participants aged 12 years and older over time. As such, the aims of the PATH Study centre on research questions that enhance the evidence base needed to inform FDA's tobacco regulatory activities. Given its research purposes, the PATH Study complements and is complemented by national cross-sectional surveillance studies, such as the Tobacco Use Supplement to the Current Population Survey, the NSDUH, the National Health Interview Survey, the National Youth Tobacco Survey and the MTF, ${ }^{19-21}$ which estimate the national prevalence of a variety of health behaviours and conditions, including tobacco use. The PATH Study's research focus is most clearly illustrated by its eight overarching objectives (box 1), which guides the questionnaire development. With each wave of follow-up, the PATH Study can examine its objectives, iteratively and cumulatively, to generate a broad body of knowledge about tobacco product use in the USA.

\section{THE PATH STUDY DESIGN FEATURES}

The PATH Study is a national longitudinal cohort study of 45971 adults and youth 12 years of age and older. Wave 1 data collection began on 12 September 2013 and ended on 14

\section{Box 1 The PATH Study primary objectives}

1. Identify and explain between-person differences and within-person changes in tobacco-use patterns.

2. Identify between-person differences and within-person changes in risk perceptions regarding harmful and potentially harmful constituents, new and emerging tobacco products, tobacco design features, packaging and labelling and identify other factors that may affect use.

3. Characterise the natural history of tobacco dependence, cessation and relapse.

4. Assess between-person differences and within-person changes over time in health conditions potentially related to tobacco use, including markers of exposure and tobacco-related disease processes identified from the collection and analysis of biospecimens.

5. Assess the association between implementing the TCA and tobacco product use, risk perceptions, attitudes, use patterns, cessation outcomes and tobacco-related intermediate end points.

6. Assess between-person differences and within-person changes over time in attitudes, behaviours, exposures to tobacco products and related biomarkers among and within population subgroups defined by race/ethnicity, gender, age and risk factors.

7. Assess and compare former and never users of tobacco products for between-person differences and within-person changes over time in relapse and uptake, risk perceptions and indicators of tobacco product exposure and disease processes.

8. Use the PATH Study as a basis for screening respondents for participation in small-scale research studies.
December 2014. Wave 1 of the PATH Study included the following features:

1. An initial, nationally representative household sample of 45971 persons (including 32320 adults aged 18 and older and 13651 youths aged 12-17);

2. The oversampling of tobacco users, young adults aged 18-24 and African-Americans (due to higher rates of menthol cigarette use compared to those other races/ethnic groups);

3. The use of Audio Computer-Assisted Self-Interviewing and Computer-Assisted Personal Interviewing administered questionnaires and of various paper data collection forms;

4. Bilingual (English and Spanish) field interviewers and versions of questionnaires and other survey materials for participants;

5. The Wave 1 collection of biospecimens from adults who consent to provide them, with urine and blood requested from all adults during the entire field period and buccal cells requested from adults during a portion of the field period.

Development of the Wave 1 questionnaires for the PATH Study was guided by the study objectives in box 1 . Items were broadly categorised into three areas: (1) outcome measures, such as tobacco use variables and health-related outcomes, which include intermediate outcomes hypothesised to be in a causal pathway for more distal behavioural or health outcomes; (2) measures that have potential relevance for regulatory decisions; and (3) control or moderating measures necessary to understand factors associated with changes in tobacco use and health outcomes, such as demographics and peer influences. The PATH Study adapted many items from well-established existing national surveys. For example, items about use of various tobacco products were adapted from the Tobacco Use Supplement to the Current Population Survey items on cigarette smoking to assess the full array of tobacco products. $^{22}$ Nicotine dependence items in the PATH Study's questionnaires were based in part on the National Epidemiological Survey on Alcohol and Related Conditions Survey. ${ }^{23}$ Questions on physical health end points were drawn from the National Health and Nutrition Examination Survey (NHANES), including, in some cases, adapting the question stems of the NHANES for items on health conditions that are not asked about in the NHANES. ${ }^{24}$ Other health-related items in the PATH Study questionnaires came from validated screeners including the Global Appraisal of Individual Needs (GAIN) and Patient Reported Outcomes Measurement Information System (PROMIS). ${ }^{25-27}$ The PATH Study questionnaires also included items from international, state and privately funded tobacco surveillance surveys, such as the ITC Project, the Visual Media Influences on Adolescent and Young Adult Smoking Behaviour surveys and the Minnesota Adult Tobacco Survey. In addition, the PATH Study questionnaires were tailored to take advantage of the Audio Computer-Assisted Self-Interviewing (ACASI) and Computer-Assisted Personal Interviewing (CAPI) administrations. For example, images of tobacco products were used to help the respondent identify which products they used, as was extensive use of tailored question wording based on responses to previous items. Selected study materials can be found at http://doi.org/10.3886/ICPSR36231.

\section{Sample design and weighting}

The population of interest for the PATH Study in Wave 1 is the 2013 civilian, non-institutionalised US population 12 years of age and older. A four-stage stratified area probability sample design was used, with a two-phase design for sampling the adult cohort at the final stage. A detailed description of the design can be found in the User's Guide to the restricted use files 
(RUFs), available at http://doi.org/10.3886/ICPSR36231. At the first stage, a stratified sample of 156 geographical primary sampling units (PSUs) was selected, in which a PSU is a county or group of counties. Within each selected PSU, the second stage formed and sampled smaller geographical segments. The thirdstage sampled residential addresses in the US Postal Service Computerised Delivery Sequence Files, with coverage enhancement procedures used to include addresses not listed in those files. $^{28}$ The fourth stage selected persons from the sampled households. A roster of all members in the sampled household was constructed by interviewing one adult household member (referred to as the household informant) to list the household members and collect information about each one for use in sampling the three groups of interest:

- Adults (up to two adults per household);

- Children aged 12-17 (referred to as 'youth', generally up to two per household);

- Children aged 9-11 (referred to as 'shadow youth', generally up to two per household) to be enrolled in the youth cohort in later waves of the study on reaching 12 years of age. ${ }^{\text {ii }}$

Owing to possible misreporting by the household informant, a two-phase sampling procedure was used to select adults within sampled households. The first phase used higher selection probabilities for young adults, African-Americans and tobacco users, based on the information provided by the household informant. The second phase of selection relied on the more accurate selfreported age, race and tobacco use status, obtained by interviewing the individuals sampled at the first phase and corrected for errors in those classifications from the household respondent.

Analysis of data from complex sample designs, such as the design used for the PATH Study, involves weighting to compensate for different probabilities of selection, non-response and possible deficiencies in the sampling frame (eg, under-coverage of certain population groups). Variance estimation procedures are also required to account for sampling design factors such as stratification and clustering. The Wave 1 weight for a PATH Study respondent is a function of (1) the inverse of the probability that a person is selected to be in the PATH Study sample; (2) factors that adjust for non-response; and (3) factors that calibrate estimates from the sample to quantities known from the 2010 US Decennial Census and the 2013 American Community Survey. The PATH Study design oversamples tobacco users, young adults (aged 18-24) and African-American adults (due to higher rates of menthol cigarette use compared to those of other races). The weights adjust for this oversampling when estimating population quantities. The weight for a survey respondent may be thought of as the number of persons in the population represented by that respondent. All estimates from the PATH Study should be calculated using weights.

As future waves of data are collected, longitudinal weights will be created that allow data from multiple time periods to be analysed and that will account for attrition. Longitudinal data gathered by the study can be used to explore mediational models assessing temporal relationships between 'exposure' and 'outcome' variables.

Replicate weights, calculated using Fay's variant of balanced repeated replication, ${ }^{29}$ are provided for the calculation of SEs.

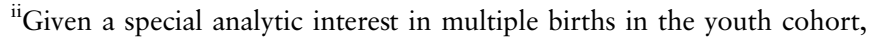
sampling procedures were modified to allow for additional youth when households containing multiple births among youths and/or shadow youths were encountered.
}

The weights and replicate weights enable the data to be analysed using standard software packages that support survey data analysis.

Table 1 provides the Wave 1 sample size for the entire sample (by demographic subgroups) and for adults who provided various biospecimens. The PATH Study sample sizes were determined so that estimates of proportions would have relative SEs $<5 \%$ for national estimates and for predefined subgroups defined by race/ethnicity, gender and age. Table 2 provides sample sizes by Wave 1 current use status of different tobacco products, where adult current use is defined, among those who reported having 'ever' used a specific product, as those who reported currently using that product 'every day' or 'some days'. For youth, current use of a product is defined as any use in the past 30 days.

\section{Data collection protocol and tools}

The Westat Institutional Review Board approved the study design and protocol to safeguard the rights, welfare and wellbeing of all humans involved in this study; the Office of Management and Budget approved the data collection. The PATH Study protocol called for in-person collection of all data and biospecimens for Wave 1. This protocol included four main data collection components: (1) a CAPI household screening questionnaire administered by an interviewer, (2) selfadministered ACASI questionnaires for the adult and youth interviews, (3) a CAPI questionnaire administered by an interviewer to the parent or guardian of each youth and (4) collection of biospecimens from consenting adults. At a minimum, completion of an individual adult or youth Wave 1 interview was required for enrolment of a sampled individual in the study.

\section{CAPI household screening questionnaire}

The process of sampling persons began with a household screening interview of an adult household member aged 18 or older, who provided information that was used to select a probability sample of up to two adults and two youths ${ }^{\text {iii }}$ per eligible household. The screener enumerated the entire household to collect information on the age, race, active military service status and tobacco use for each adult household member.

\section{ACASI questionnaire for adult interview and biospecimen procedures}

After administering the household screener, the field interviewer asked each sampled adult who was available to complete the interview to (1) provide informed consent for the interview; (2) complete the Adult Interview by ACASI, including providing contact information; (3) review and complete the consent for biospecimen collection; (4) provide the biospecimens (buccal cell sample and/or urine); and (5) schedule a follow-up appointment for a phlebotomist to collect a blood sample. If a sampled adult was unavailable for the interview following the screener, the field interviewer scheduled an appointment for a return visit.

The first part of the Adult Interview was the second-phase individual screener. Responses by the sampled adult to these items may have confirmed or contradicted the information obtained during the household screener. In some cases, an adult sampled as the result of the household screener was not

\footnotetext{
iii Up to two youths were sampled per household unless a household included multiple births, in which case additional youth could be selected.
} 
Table 1 Wave 1 sample sizes for completed interviews and collected biospecimen types, overall and by selected demographic subgroups

\begin{tabular}{|c|c|c|c|c|c|c|c|}
\hline & \multicolumn{7}{|c|}{ Wave 1 sample size } \\
\hline & \multirow[b]{3}{*}{ Youth interview } & \multicolumn{6}{|l|}{ Adults } \\
\hline & & \multirow[b]{2}{*}{ Interview } & \multicolumn{5}{|c|}{ Collected biospecimens } \\
\hline & & & Urine & Blood & Buccal* & Urine and blood & All 3* \\
\hline Overall & 13651 & 32320 & 21801 & 14520 & 12543 & 13856 & 6950 \\
\hline \multicolumn{8}{|l|}{ Age (years) } \\
\hline $12-17$ & $13651 \dagger$ & & & & & & \\
\hline $18-24$ & & 9112 & 6459 & 3886 & 3866 & 3659 & 1839 \\
\hline $25-34$ & & 6338 & 4380 & 2826 & 2470 & 2712 & 1315 \\
\hline $35-44$ & & 4931 & 3361 & 2178 & 1790 & 2102 & 1006 \\
\hline $45-54$ & & 4846 & 3164 & 2276 & 1859 & 2181 & 1138 \\
\hline $55-64$ & & 3972 & 2561 & 1915 & 1458 & 1831 & 943 \\
\hline $65+$ & & 3110 & 1873 & 1438 & 1100 & 1370 & 709 \\
\hline \multicolumn{8}{|l|}{ Gender } \\
\hline Man & 6971 & 16309 & 10763 & 6920 & 6155 & 6633 & 3367 \\
\hline Woman & 6641 & 15982 & 11025 & 7594 & 6381 & 7217 & 3580 \\
\hline \multicolumn{8}{|l|}{ Race/ethnicity } \\
\hline White, non-Hispanic & 6478 & 19299 & 12818 & 8895 & 7474 & 8509 & 4312 \\
\hline Black, non-Hispanic & 1801 & 4496 & 3168 & 1913 & 1758 & 1815 & 872 \\
\hline Hispanic & 3880 & 5536 & 3870 & 2452 & 2241 & 2335 & 1216 \\
\hline American Indian or Alaska Native, non-Hispanic & 70 & 195 & 138 & 102 & 86 & 93 & 44 \\
\hline Asian/Native Hawaiian or Other Pacific Islander, non-Hispanic & 394 & 966 & 543 & 299 & 290 & 285 & 126 \\
\hline Multiple races, non-Hispanic & 767 & 1269 & 929 & 655 & 511 & 623 & 287 \\
\hline
\end{tabular}

Table 2 Wave 1 sample sizes for completed interviews and collected biospecimen type by Wave 1 current tobacco-use status

\begin{tabular}{|c|c|c|c|c|c|c|c|}
\hline \multirow[b]{4}{*}{ Tobacco product use } & \multicolumn{7}{|c|}{ Wave 1 sample size } \\
\hline & \multirow[b]{3}{*}{ Youth interview } & \multicolumn{6}{|l|}{ Adults } \\
\hline & & \multirow[b]{2}{*}{ Interview } & \multicolumn{5}{|c|}{ Collected biospecimens } \\
\hline & & & Urine & Blood & Buccal* & Urine and blood & All 3* \\
\hline Any current tobacco use & $1151 \dagger$ & $17732 \ddagger$ & 12533 & 8422 & 7364 & 8020 & 4086 \\
\hline Any current cigarette use & 634 & 13529 & 9760 & 6679 & 5753 & 6352 & 3268 \\
\hline Any current smokeless use, excluding snus & 180 & 1873 & 1319 & 848 & 734 & 825 & 426 \\
\hline Any current e-cigarette use & 418 & 3642 & 2666 & 1836 & 1573 & 1739 & 897 \\
\hline Any current cigarillo use & 293 & 2969 & 2310 & 1525 & 1355 & 1464 & 735 \\
\hline Any current traditional cigar use & 92 & 2586 & 1769 & 1173 & 1047 & 1124 & 575 \\
\hline Any current hookah use & 226 & 3134 & 2306 & 1414 & 1401 & 1336 & 694 \\
\hline Any current filtered cigar use & 67 & 1295 & 999 & 689 & 629 & 658 & 383 \\
\hline Any current pipe use & 41 & 709 & 518 & 354 & 300 & 339 & 174 \\
\hline Any current snus use & 61 & 479 & 366 & 256 & 227 & 247 & 132 \\
\hline Any current dissolvable & 3 & 67 & 52 & 31 & 33 & 30 & 14 \\
\hline Any current bidi use & 6 & & & & & & \\
\hline Any current kretek use & 11 & & & & & & \\
\hline No current tobacco use & 11815 & 13902 & 8904 & 5862 & 4986 & 5611 & 2755 \\
\hline
\end{tabular}

selected to continue with the Adult Interview based on the second-phase individual screener. Otherwise, the sampled adult completed the interview, including providing contact information for follow-up in future waves. The Wave 1 Adult
Interview averaged $\sim 60$ min. After completing the interview, the adult respondent was asked to provide consent for the collection of biospecimens. Each adult received a $\$ 35$ incentive for completing the interview. 


\section{CAPI questionnaire for parent interview}

After the household screener, the parent or guardian of a selected youth was asked to provide (1) parental permission for the youth's participation and (2) consent for a short parent interview. At this point, the field interviewer administered the CAPI parent interview. In some instances, more than one youth may have been sampled as a result of the household screener and the parent was asked for information on each sampled youth. A parent received a $\$ 10$ incentive for completing each interview.

\section{ACASI questionnaire for youth interview}

After obtaining parental permission for a selected youth, the field interviewer asked the youth for his or her assent to complete the ACASI questionnaire. The Wave 1 Youth Interview averaged $\sim 35 \mathrm{~min}$. Each youth received a $\$ 25$ incentive for completing the interview.

\section{Biospecimen collection-adults}

Field interviewers asked adults who had completed the interview to provide consent for the collection of up to three biospecimens, described below. To aid the interpretation of biomarker results, respondents were also asked to record use of all nicotine-containing products over a 3-day period prior to the time of any biospecimen collection.

\section{Urine and buccal cells}

Field interviewers collected urine and buccal cell samples from consenting adults. Urine was collected over the full Wave 1 data collection period. Owing to competing priorities and limited funding, buccal cells were collected for a limited time, from 12 September 2013 to 18 May 2014. Respondents who provided biospecimens received a $\$ 25$ incentive for participating in the buccal cell and urine sample component of the study, regardless of whether they provided one or both biospecimens.

\section{Urine}

Full-void urine specimens were self-collected by consenting participants in a $500 \mathrm{~mL}$ polypropylene container (PN 6542; Globe Scientific), which was immediately placed in a custom Crēdo Cube shipper (Series 4-496; Minnesota Thermal Science) certified to hold contents between $2^{\circ} \mathrm{C}$ and $8^{\circ} \mathrm{C}$ for at least 72 hours and shipped overnight to the PATH Study biorepository. Each specimen was processed at the biorepository and divided into aliquots totalling to up to $50 \mathrm{~mL}$ for each participant. Aliquots were placed in long-term storage at $-80^{\circ} \mathrm{C}$.

\section{Buccal cells}

Buccal cells were collected following a method for obtaining high-quality RNA from buccal mucosa. ${ }^{30}$ Cells were selfcollected by consenting participants using a custom plastic collection tool manufactured for the PATH Study by Piedmont Plastics. Collected cells were immediately immersed in $1.5 \mathrm{~mL}$ RNAprotect Cell Reagent (76 526, Qiagen) in a $2.0 \mathrm{~mL}$ cryovial and placed in the same Crēdo Cube shipper as the urine (when both were collected). The phial was also placed in long-term storage at $-80^{\circ} \mathrm{C}$.

\section{Blood}

Blood was collected from consenting adults by a phlebotomist, who visited the home at a time scheduled by the field interviewer. The phlebotomist administered blood donation suitability exclusion questions and a brief set of questions on the respondent's use of products that contain nicotine over a 3-day period prior to blood collection. Respondents received a $\$ 25$ incentive for participating in the blood sample component of the study.

The tubes used to collect blood included one $2.7 \mathrm{~mL}$ blue top citrate (BD Vacutainer 363083), two $10.0 \mathrm{~mL}$ red top serum (BD Vacutainer 367820), two $10.0 \mathrm{~mL}$ lavender top EDTA (BD Vacutainer 366643) and one $2.5 \mathrm{~mL}$ PAXgene (BD Vacutainer 762165). Collected blood was immediately placed in a Credo Cube shipper (Series 4-248; Minnesota Thermal Science) and shipped overnight to the PATH Study biorepository. The citrate tube was centrifuged and separated into plasma and pellet (white cell count and red blood cell) fractions. The serum tubes were centrifuged, and the resulting serum fraction was divided into aliquots. The EDTA tubes were centrifuged and separated into plasma, buffy coat and red blood cell fractions. Plasma was divided into aliquots. All blood aliquots were placed in longterm storage at $-80^{\circ} \mathrm{C}$. The PAXgene tube was stored without processing at $-20^{\circ} \mathrm{C}$.

\section{Biospecimen analysis}

A portion of the stored biospecimens provided by a subsample of 11500 adult respondents are being analysed by the Centers for Disease Control and Prevention (CDC) Division of Laboratory Sciences. The sampling scheme used to constitute the subsample sought to obtain a diverse mix of tobacco product use patterns by sampling from mutually exclusive user groups including exclusive current cigarette users, current users of other tobacco products, experimental-only users of any tobacco product, former users of tobacco products and never users. Laboratory analyses by CDC focus on biomarkers of tobacco exposure (eg, nicotine, cotinine and other nicotine metabolites, minor tobacco alkaloids, tobacco-specific nitrosamines, volatile organics, polycyclic aromatic hydrocarbons, heavy metals and speciated arsenic) and disease (eg, F2-isoprostane and C reactive protein) associated with the use of tobacco products. Biospecimens provided by the rest of the adult cohort remain in storage for analysis at a later time.

\section{Quality control of questionnaire and biospecimen collection}

To maximise the Wave 1 response rate and quality of data and biospecimens, the PATH Study employed a large team of field interviewers, field supervisors and phlebotomists who received in-depth training in the procedures of administering each step of the protocol, including the consent process, data and biospecimen collection, follow-up and the importance of working as a team. Interviewers were required to complete a week-long training and obtain certification before beginning fieldwork. Experienced phlebotomists were also trained on the PATH Study protocol to collect blood samples in the participants' homes.

The PATH Study provided incentives to thank respondents for participating in the study; used bilingual interviewers and Spanish versions of the questionnaires and study materials; sent an advance letter to the household at each sampled address prior to the screener visit to inform its residents about the PATH Study, including its purpose and the voluntary and confidential nature of the information collected; created a PATH Study website to provide up-to-date information about the study and widely disseminated a toll-free telephone number that respondents or others who had questions about the study could call. PATH Study letters were also developed for respondents or sampled persons who appeared reluctant or not interested in participating in the study. 


\section{RESPONSE RATES}

The PATH Study computed the response rates for the Wave 1 data collection in accordance with the AAPOR guidelines. ${ }^{31}$ The weighted response rate for the household screener was $54.0 \%$. Among households that were screened, the overall weighted response rate was $74.0 \%$ for the Adult Interview and $78.4 \%$ for the Youth Interview. Among those who completed the Adult Interview, the weighted response rate was $63.6 \%$ for providing a urine sample and $43.0 \%$ for providing a blood sample.

A nonresponse bias analysis for Wave 1 has been performed and can be found at http://doi.org/10.3886/ICPSR36231. Briefly, the weighted distributions of demographic characteristics for the Wave 1 adult sample are similar to those from the 1-year 2013 American Community Survey (ACS) for gender, age and education although there are slightly higher weighted percentages of whites, blacks and Hispanics in the PATH sample compared to ACS. The weighted demographic characteristics for the Wave 1 youth sample are similar to the 1-year 2013 ACS for age and gender and the weighted percentages of Hispanic, non-Hispanic white and non-Hispanic other youth are all within 0.2 percentage points of the ACS values.

\section{CURRENT AND FUTURE DIRECTIONS OF THE PATH STUDY}

The currently funded project period for the PATH Study includes the completion of three annual waves of behavioural data and biospecimen collection. Data collection for Wave 2 and Wave 3 began October 2014 and October 2015, respectively. ${ }^{\text {iv }}$ NIDA and CTP are committed to maximising the research value of the PATH Study by delivering its behavioural and biological data to a designated repository for researchers to access and use, first by way of RUFs and then, following a period of data disclosure analysis, by way of public use files. As of January 2016, access to the RUF can be requested by the research community at http://doi. org/10.3886/ICPSR36231, and subsequent waves of behavioural data will be placed in the repository $\sim 1$ year following the completion of each wave. Biospecimen data will be available at a later time when laboratory analyses are completed. Over time, researchers will be able to access and merge various files from this library for each wave to inform their research studies. Cumulative data generated by the PATH Study on tobacco use and health conditions among the US population will, over time, provide valuable information to enhance the evidence base that informs FDA's regulatory mission and advances knowledge of the determinants and health impacts of tobacco product use.

\section{CONCLUSIONS}

In 2009, the TCA was signed into law, creating the CTP within the FDA and giving the agency broad regulatory authority over the manufacturing, marketing and distribution of tobacco products for the protection of public health. With support by FDA, through NIH, the PATH Study is designed to enhance the evidence base that informs CTP's regulatory mission and to advance tobacco product use knowledge for the broader scientific community. Its breadth, scope and methodological rigour give the PATH Study a strong foundation to achieve its objectives and serve as a valuable resource for FDA's regulatory decisions in the years to come.

\footnotetext{
${ }^{\text {iv }}$ The field period for each wave is $\sim 1$ year. Subject to future funding, NIDA and CTP expect to continue to follow-up the PATH Study cohort to meet the PATH Study's eight objectives. Study procedures for future waves of data collection will be available on the Office of Management and Budget website.
}

\section{What this paper adds}

- In 2009 the Family Smoking Prevention and Tobacco Control Act was signed into law giving the Food and Drug Administration (FDA) broad regulatory authority over the manufacturing, marketing and distribution of tobacco products.

- The Population Assessment of Tobacco and Health (PATH) Study was launched in September 2013 to generate longitudinal epidemiologic data on tobacco use behaviors and health among the US population to inform and to monitor the impact of FDA's tobacco regulatory actions.

- Information presented in this paper describes the methods and conceptual framework for Wave 1 of the PATH Study data collection, which is intended to serve as the basis for subsequent papers using the PATH Study data.

Authors Andrew Hyland ${ }^{1}$, Bridget K Ambrose ${ }^{2}$, Kevin P Conway ${ }^{3}$, Nicolette Borek², Elizabeth Lambert ${ }^{3}$, Charles Carusi ${ }^{4}$, Kristie Taylor ${ }^{4}$, Scott Crosse ${ }^{4}$, Geoffrey T Fong ${ }^{5,6}, K_{\text {Michael Cummings }}^{7}$, David Abrams ${ }^{8}$, John P Pierce ${ }^{9}$, James Sargent ${ }^{10}$, Karen Messer ${ }^{9}$, Maansi Bansal-Travers ${ }^{1}$, Ray Niaura ${ }^{8}$, Donna Vallone ${ }^{8}$, David Hammond $^{6}$, Nahla Hilmi ${ }^{3}$, Jonathan Kwan ${ }^{2}$, Andrea Piesse ${ }^{4}$, Graham Kalton ${ }^{4}$, Sharon Lohr ${ }^{4}$, Nick Pharris-Ciurej ${ }^{2}$, Victoria Castleman ${ }^{4}$, Victoria R Green ${ }^{3,13}$, Greta Tessman ${ }^{2}$, Annette Kaufman ${ }^{11}$, Charles Lawrence ${ }^{4}$, Dana M van Bemmel ${ }^{2}$, Heather L Kimmel $^{3}$, Ben Blount ${ }^{12}$, Ling Yang ${ }^{2}$, Barbara O'Brien ${ }^{4}$, Cindy Tworek ${ }^{2}$, Derek Alberding' ${ }^{2}$, Lynn C Hull ${ }^{2}$, Yu-Ching Cheng ${ }^{2}$, David Maklan ${ }^{4}$, Cathy L Backinger ${ }^{2}$, Wilson M Compton ${ }^{3}$

\section{Affiliations}

${ }^{1}$ Roswell Park Cancer Institute, Buffalo, New York, USA

${ }^{2}$ Center for Tobacco Products, US Food and Drug Administration, Silver Spring, Maryland, USA

${ }^{3}$ National Institute on Drug Abuse, National Institutes of Health, US Department of Health and Human Services, Bethesda, Maryland, USA

${ }^{4}$ Westat, Rockville, Maryland, USA

${ }^{5}$ University of Waterloo, Waterloo, Ontario, USA

${ }^{6}$ Ontario Institute for Cancer Research, Toronto, Ontario, USA

${ }^{7}$ Medical University of South Carolina, Charleston, South Carolina, USA

${ }^{8}$ Schroeder Institute for Tobacco Research and Policy Studies, Truth Initiative,

Washington, District of Columbia, USA; The Johns Hopkins Bloomberg School of

Public Health, Baltimore, Maryland, USA

${ }^{9}$ University of California at San Diego, San Diego, California, USA

${ }^{10}$ Dartmouth College, Hanover, New Hampshire, USA

${ }^{11}$ National Cancer Institute, National Institutes of Health, US Department of Health and Human Services, Bethesda, Maryland, USA

${ }^{12}$ CDC National Center for Environmental Health, US Department of Health and Human Services, Atlanta, Georgia, USA

${ }^{13}$ Kelly Government Solutions, Rockville, Maryland, USA

Acknowledgements The authors are grateful for the participants who have volunteered to be a part of the PATH Study and make this work possible. The PATH Study would also not be possible without the scientific collaboration of NIH/NIDA, FDA/CTP, CDC and Westat and Partner Organizations, and the authors acknowledge the following PATH Study team members who were responsible for the conceptualisation, development, implementation and/or oversight of PATH Study Wave 1 operations including, but not exclusively, Genevieve Vullo from the $\mathrm{NIH} /$ NIDA; Corinne Husten and Allison Hoffman from the FDA/CTP and Tammy Cook, Nina Hamburg, Marsha Hasson, Ellen Herbold, Mary Laidlaw, Lucy Leuchtenburg, Jeffrey Phillips and Vivek Rau from Westat.

Contributors The PATH Study is a large and complex study with integral input provided for the planning, conduct and reporting of the study from each author as noted below. KEY: $P=$ contributed to Planning the study; $C=$ contributed to Conducting the study; $R=$ contributed to Reporting on the study; $G=$ responsible as Guarantor for the paper. Andrew Hyland (PCRG); Bridget K Ambrose (PR); Kevin P Conway (PCRG); Nicolette Borek (PCRG); Elizabeth Lambert (PR); Charles Carusi (PCR), Kristie Taylor (PCR); Scott Crosse (PCR); Geoffrey T Fong (PR); K Michael Cummings (PR); David Abrams (PR); John Pierce (PR); James Sargent $(\mathrm{PR})$; Karen Messer (PR); Maansi Bansal-Travers (PR); Ray Niaura (PR); Donna Vallone (PR); David Hammond (PR); Nahla Hilmi (PR); Jonathan Kwan (PR); 
Andrea Piesse (PCR); Graham Kalton (PCR); Sharon Lohr (PCR); Nick Pharris-Ciurej (PR); Victoria Castleman (PCR); Victoria R Green (PR); Greta Tessman (PR); Annette Kaufman (PR); Charles Lawrence (PCR); Dana M van Bemmel (PR); Heather L Kimmel (PR); Ben Blount (PR); Ling Yang (PR); Barbara O'Brien (PCR); Cindy Tworek (PR); Derek Alberding (PR); Lynn C Hull (PR) Yu-Ching Cheng (PR); David Maklan (PCR); Cathy L Backinger (PCRG) and Wilson M Compton (PCRG).

Funding This work was supported with Federal funds from the National Institute on Drug Abuse, National Institutes of Health and the Food and Drug Administration, Department of Health and Human Services, under a contract to Westat (Contract No. HHSN271201100027C).

Disclaimer The views and opinions expressed in this presentation are those of the authors only and do not necessarily represent the views, official policy or position of the US Department of Health and Human Services or any of its affiliated institutions or agencies.

Competing interests KMC has received grant funding from Pfizer, to study the impact of a hospital-based tobacco cessation intervention. He also receives funding as an expert witness in litigation filed against the tobacco industry. WC reports long-term stock holdings in General Electric Company, 3M Companies and Pfizer, all unrelated to this manuscript. GTF has received funding as an expert witness and consultant to governments whose policies are being challenged by the tobacco industry. DH receives funding as an expert witness in litigation filed against the tobacco industry.

Ethics approval Westat's institutional review board approved this study.

Provenance and peer review Not commissioned; externally peer reviewed.

Data sharing statement Further information regarding sharing of the data and access to the restricted use file (RUF) is based on request through the National Addiction and HIV Data Archive Program, a program supported by the National Institute on Drug Abuse, part of the National Institutes of Health. http://doi.org/10. 3886/ICPSR36231.

\section{REFERENCES}

1 US Department of Health EaW. Smoking and health: report of the Advisory Committee to the Surgeon General of the Public Health Service. Report No.: PHS Publication No. 1103. Washington DC: US Department of Health, Education, and Welfare, Public Health Service, 1964.

2 US Department of Health and Human Services. The health consequences of smoking -50 years of progress: a report of the Surgeon General. Atlanta, GA: US Department of Health and Human Services, Centers for Disease Control and Prevention, National Center for Chronic Disease Prevention and Health Promotion, Office on Smoking and Health, 2014.

3 Rostron BL, Chang CM, Pechacek TF. Estimation of cigarette smoking-attributable morbidity in the United States. JAMA Intern Med 2014;174:1922-8.

4 Substance Abuse and Mental Health Services Administration. Results from the 2013 National Survey on Drug Use and Health: summary of national findings. Report No.: NSDUH Series H-48, HHS Publication No. (SMA) 14-4863. Rockville, MD: Substance Abuse and Mental Health Services Administration, 2014.

5 Arrazola RA, Singh T, Corey CG, et al. Tobacco use among middle and high school students-United States, 2011-2014. MMWR Morb Mortal Wkly Rep 2015;64:381-5.

6 Miech RA, Johnston LD, O'Malley PM, et al. E-cigarettes surpass tobacco cigarettes among teens. National Press Release 16 December 2014:1-16.

7 Family Smoking Prevention and Tobacco Control Act, H.R. 1256.6-16, 111th Congress. 2009.

8 Cobb C, Villanti AC, Graham AL, et al. Markov modeling to estimate the population impact of emerging tobacco products: a proof-of-concept study. Tob Regul Sci 2015:1:129-41.
9 Vugrin ED, Rostron BL, Verzi SJ, et al. Modeling the potential effects of new tobacco products and policies: a dynamic population model for multiple product use and harm. PLoS One 2015;10:e0121008.

10 Levy DT, Cummings KM, Villanti AC, et al. A framework for evaluating the public health impact of e-cigarettes and other vaporized nicotine products. Addiction 2017; 112:8-17.

11 Giovino GA. Epidemiology of tobacco use in the United States. Oncogene 2002:21:7326-40.

12 Fong GT, Cummings KM, Borland R, et al. The conceptual framework of the International Tobacco Control (ITC) Policy Evaluation Project. Tob Control 2006;15 (Suppl 3):iii3-11.

13 International Agency for Research on Cancer. IARC handbooks of cancer prevention, tobacco control, Vol. 12: methods for evaluating tobacco control policies. Lyon, France: International Agency for Research on Cancer, 2008.

14 Abrams DB. Nicotine addiction: paradigms for research in the 21st century. Nicotine Tob Res 1999;1(Suppl 2):S211-15.

15 Abrams DB. Transdisciplinary paradigms for tobacco prevention research. Nicotine Tob Res 1999;1(Suppl 1):S15-23.

16 Abrams DB, Leslie F, Mermelstein $\mathrm{R}$, et al. Transdisciplinary tobacco use research. Nicotine Tob Res 2003;5(Suppl 1):S5-10.

17 Abrams DB. Applying transdisciplinary research strategies to understanding and eliminating health disparities. Health Educ Behav 2006;33:515-31.

18 Mabry PL, Olster DH, Morgan GD, et al. Interdisciplinarity and systems science to improve population health: a view from the NIH Office of Behavioral and Social Sciences Research. Am J Prev Med 2008;35:S211-24.

19 Ryan H, Trosclair A, Gfroerer J. Adult current smoking: differences in definitions and prevalence estimates_-NHIS and NSDUH, 2008. J Environ Public Health 2012:2012:918368.

20 Soulakova J, Davis WW, Hartman A, et al. The impact of survey and response modes on current smoking prevalence estimates using TUS-CPS: 1992-2003. Surv Res Methods 2009;3:123-37.

21 Substance Abuse and Mental Health Services Administration. Comparing and evaluating youth substance use estimates from The National Survey on Drug Use and Health and other surveys. Report No.: HHS Publication No. SMA 12-4727, Methodology Series M-9. Rockville, MD: Substance Abuse and Mental Health Services Administration, 2012

22 US Department of Commerce CB. National Cancer Institute-sponsored Tobacco Use Supplement to the Current Population Survey (2010-2011). 2012. http:// appliedresearch.cancer.gov/tus-cps. Technical documentation. http://www.census. gov/cps/methodology/techdocs html

23 Grant BF, Dawson DA. Introduction to The National Epidemiological Survey on Alcohol and Related Conditions. Alcohol Res Health 2006:29:74-9.

24 Centers for Disease Control and Prevention. National Health and Nutrition Examination Survey. 2015. http://www.cdc.gov/nchs.nhanes.htm

25 Dennis ML, Chan YF, Funk RR. Development and validation of the GAIN Short Screener (GSS) for internalizing, externalizing and substance use disorders and crime/violence problems among adolescents and adults. Am J Addict 2006;15 (Suppl 1):80-91.

26 Chestnut Health Systems GCC. GAIN: Global Appraisal of Individual Needs Coordinating Center. 2014 (cited 3 December 2014). http://www.gaincc.org

27 U.S.Department of Health and Human Services. PROMIS: Patient-Reported Outcomes Measurement Information System. 2014 (cited 3 December 2014). https://commonfund nih gov/promis/index

28 Kalton G, Kali J, Sigman R. Handling frame problems when address-based sampling is used for in-person household surveys. J Surv Stat Methodol 2014;2:1-22.

29 Judkins DR. Fay's method for variance estimation. J Off Stat 1990;6:223-39.

30 Spira A, Beane J, Schembri F, et al. Noninvasive method for obtaining RNA from buccal mucosa epithelial cells for gene expression profiling. Biotechniques 2004;36:484-7.

31 The American Association for Public Opinion Research. Standard definitions: final dispositions of case codes and outcome rates for surveys. 8th edn. Lenexa, KS: AAPOR, 2015. 